\title{
Allergie und Mikrobiota
}

\author{
Stellenwert mikrobieller Einflüsse bei der Genese allergischer und chronisch- \\ entzündlicher Krankheitsbilder sowie therapeutische Einflussnahme mit Mikro- \\ biologischer Therapie
}

Autoren: Susanne Schnitzer, Rainer Schmidt

\section{ZUSAMMENFASSUNG}

Noch immer wird eine allergische Reaktion als schicksalhaftes Geschehen angesehen, deren genetische Verankerung bewiesen und eine Erkrankung unvermeidbar ist. Diese Sichtweise rechtfertigt die klassischen symptomatischen Behandlungsstrategien. Systemisch gesehen entwickelt sich die allergische Reaktion jedoch aus dem immunologischen Bestreben, die menschliche Integrität gegenüber einer grundsätzlich feindlichen Umwelt zu wahren oder wiederherzustellen. Mit neuen Erkenntnissen über die menschliche Mikrobiota und das Mikrobiom sowie ihrem komplexen Zusammenspiel mit Schleimhautorgan, Immunsystem und Stoffwechsel werden Therapieansätze der Mikrobiologischen Therapie zunehmend verstanden. Kausale sowie präventive Therapieansätze für chronisch entzündliche Krankheitsbilder eröffnen sich.

\section{ABSTRACT}

An allergic reaction is still considered to be a fateful event which is firmly anchored in our genes and which inevitably causes a disease. This view justifies the classical symptomatic treatment strategies. From the systemic point of view however, the allergic reaction is the result of the immunological effort, to keep or to restore human integrity with respect to a basically hostile environment. Due to new findings concerning human microbiota and the microbiome as well as their complex interaction with the mucosal organ, immune system, and metabolism, the therapeutic approaches of the microbiological therapy are better understood. Causal as well as preventive therapeutic approaches for chronic inflammatory clinical pictures are emerging.

Keywords

Microbiological therapy, allergy, microbiota, microbium, intestine, mucous membrane, mucosa immune system.
Schlüsselwörter

Mikrobiologische Therapie, Allergie, Mikrobiota, Mikrobiom, Intestinum, Schleimhaut, Mukosa-Immunsystem

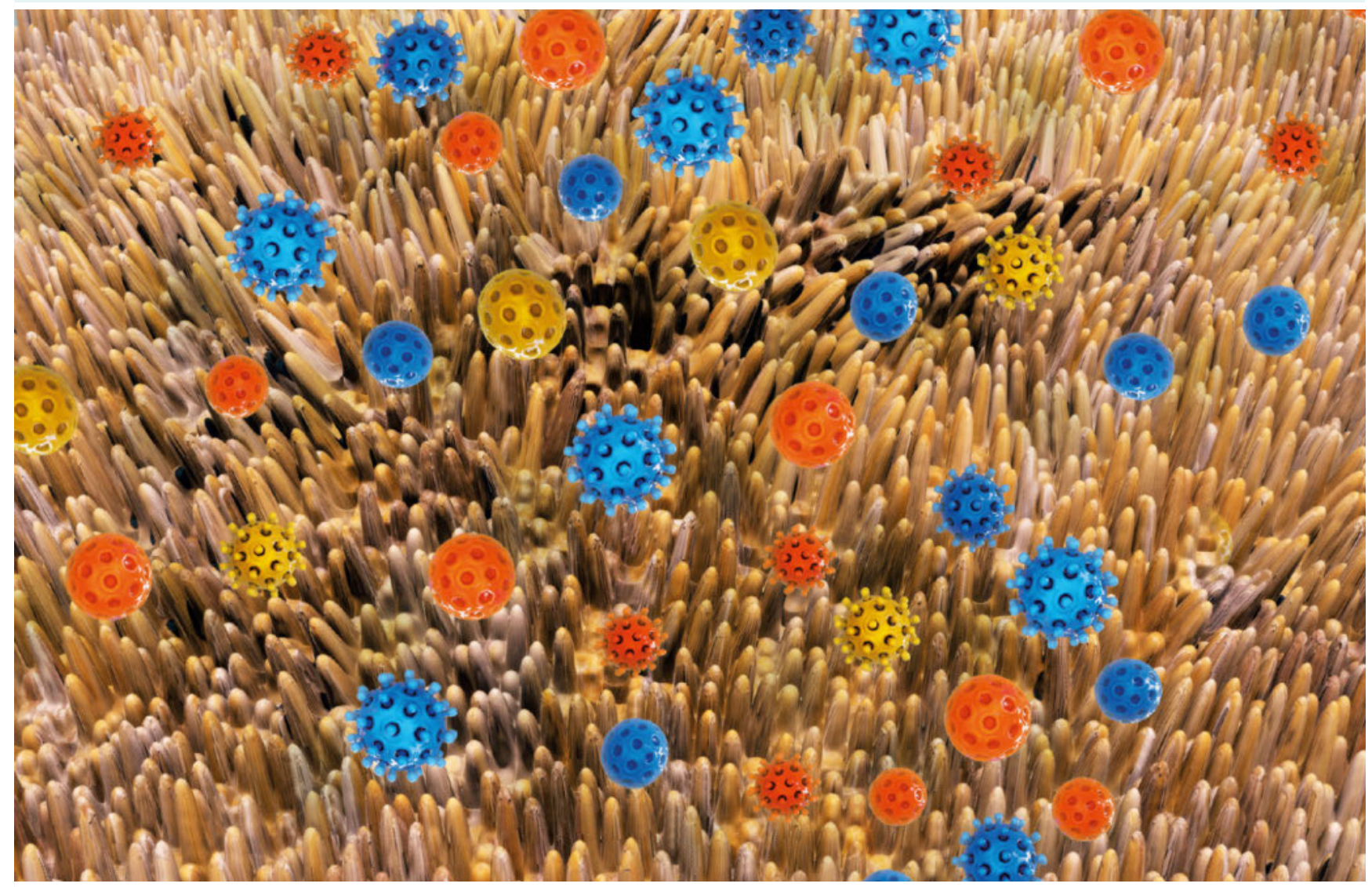

>Abb. 1 In der Darmschleimhaut befinden sich 10-100 Billionen Bakterien, die rund 400 Bakterienarten angehören. @ foliaxrender/Adobe Stock 


\section{Mikrobiota und die Mikrobiologische Therapie}

Der Begriff menschliches Mikrobiom hat sowohl in der Wissenschaft als auch in der breiten Öffentlichkeit in letzter Zeit große Aufmerksamkeit gefunden. Er bezeichnet die Gesamtheit aller Mikroorganismen, die den Menschen besiedeln. Die permanente Präsenz einer unvorstellbar großen Anzahl von Mikroorganismen ist kein Zufall und weder schlecht noch gefährlich für den Menschen, wie immer noch vielfach vermutet wird. Der Mensch ist vielmehr ein komplex regulierter Gesamtorganismus aus dem Wirtsorganismus „Mensch“ und seiner individuellen Mikrobiota. Diese „Existenz als biologisches System“ stellt keine Ausnahme dar. Vielmehr wissen wir inzwischen, dass alle Lebewesen auf der Erde, also auch alle pflanzlichen und tierischen Lebensformen, sich evolutionär in symbiotischer Lebensweise mit ihren arteigenen Mikroorganismen entwickelten. Im engeren Sinn steht der Ausdruck „Mikrobiom“ aber für die Gesamtheit aller mikrobiellen Gene bzw. für das mikrobielle Genom eines Organismus und unterscheidet sich damit vom Begriff „Mikrobiota“, der die Gesamtheit aller seiner lebenden Mikroorganismen bezeichnet.

Die Erforschung der menschlichen Mikrobiota und ihres Mikrobioms erfuhr in den letzten Dekaden durch die Nutzung moderner Sequenzierverfahren einen enormen Zuwachs an Erkenntnissen. Insbesondere die schon lange Zeit vermuteten Zusammenhänge mikrobieller Signale mit den menschlichen Regulationsebenen werden immer klarer. Entsprechend groß ist die Bedeutung der Zusammensetzung der menschlichen Mikrobiota für eine Vielzahl von Erkrankungsbildern: Entspricht ihre Zusammensetzung nicht physiologischen Mustern (quantitative und qualitative Dysbiose), fehlen dem Körper auch die zugehörigen, wichtigen Signale für seine Regulationsebenen. Es folgen Ausgleichsreaktionen, die häufig in Krankheit münden. Auf der anderen Seite ergeben sich hier bisher viel zu wenig wahrgenommene Möglichkeiten einer therapeutischen Intervention - und noch viel mehr der Prävention. Rein wissenschaftlich stecken die Möglichkeiten therapeutischer Interventionen mit Bakterien und bakteriellen Bestandteilen erst in ihren Kinderschuhen, auf empirischem und experimentellem Wissen basierend, existieren jedoch mit der sogenannten Mikrobiologischen Therapie schon seit über 100 Jahren therapeutische Herangehensweisen an eine Vielzahl von Beschwerdebildern. Insbesondere solche des atopischen Formenkreises zählen fast traditionell zu den typischen Indikationen für die Mikrobiologische Therapie: Hintergrund ist die enge Verknüpfung immunologischer Reaktionen mit der Integrität des menschlichen „Schleimhautorgans“, unserer riesigen inneren Grenzfläche zur Außenwelt. Genau hier findet das enge Zusammenspiel zwischen Mikrobiota und dem menschlichen Organismus statt. Ohne die individuelle Mikrobiota, vor allem die des Intestinums (aber auch der übrigen Schleim- hautabschnitte), kann der Mensch kein Immunsystem entwickeln. Physiologische Verhältnisse im Grenzraum, im mikrobiellen Milieu, an der Schleimhaut und dem angrenzenden Mukosa-Immunsystem (MIS) sind die maßgeblichen Stellgrößen für Gesundheit. Oder, im Falle von dysbiotischen Verhältnissen, für eine Entwicklung immunologischer Abwehrreaktionen, die gravierende Störungen nach sich ziehen können. Diese reichen von Verdauungsproblemen bis hin zu zentralnervösen Beschwerdebildern, sehr häufig aber münden sie in einer allergischen Reaktion.

Durch den Einsatz lebender probiotischer Kulturen, deren Lysate oder Stoffwechselprodukte im Rahmen der Mikrobiologischen Therapie, können bei allergischen Reaktionen in erster Linie milieustabilisierende, aber auch gezielte immunmodulierende Effekte erzielt werden. Das therapeutische Ziel ist, über eine Stabilisierung der Verhältnisse im Grenzraum und am Schleimhautorgan die überschießenden Abwehrreaktionen des MIS einzudämmen. Die Mikrobiologische Therapie stellt damit die ideale Therapieoption bei allergischen/atopischen Krankheitsbildern als Folge dysbiotischer Veränderungen im Schleimhautorgan dar, die auch präventiv vielversprechend eingesetzt werden kann.

\section{Prägung und Entwicklung des Immunsystems}

Die Entwicklung einer allergischen Reaktionsbereitschaft ist kein genetisch bestimmtes Schicksal. Ob ein Mensch zum Allergiker wird, hängt vielmehr von verschiedenen Faktoren ab, die unsere Resilienz und Toleranz gegenüber der Umwelt modulieren. Die Entwicklung und Prägung des Immunsystems wird von bestimmten Einflüssen bestimmt, die bereits intrauterin beginnen. Hier wird bereits maßgeblich die spätere „Funktionslage“ des Immunsystems bestimmt. Mindestens genauso wichtig wie die genetische Veranlagung sind dabei beispielsweise die mütterlichen immunologischen Signale (speziell: besteht bei der Mutter ebenfalls eine proinflammatorische Situation des Immunsystems? Besteht bei der Mutter eine enterale Dysbiose?), die Art der Geburt, ob das Kind gestillt oder mit Flaschennahrung gefüttert wird und eine eventuell zu frühe Beikostfütterung [1]. Auch Impfungen in diesem sensiblen Lebensabschnitt oder die Art und Weise der Behandlung früher Infekte haben aller Wahrscheinlichkeit nach größeren Einfluss auf die Entwicklung einer chronischen Entzündungsbereitschaft als bisher allgemein angenommen.

In den weiteren Lebensabschnitten rücken negative Umwelteinflüsse ins Bild, die zu einer Störung der Barrierefunktion, Veränderung des mikrobiellen Milieus, aber auch zu einer direkten Beeinflussung des Immunsystems und Stoffwechsels führen. Neben möglichen Belastungen mit Toxinen, chemischen Stoffen oder Schwermetallen muss 


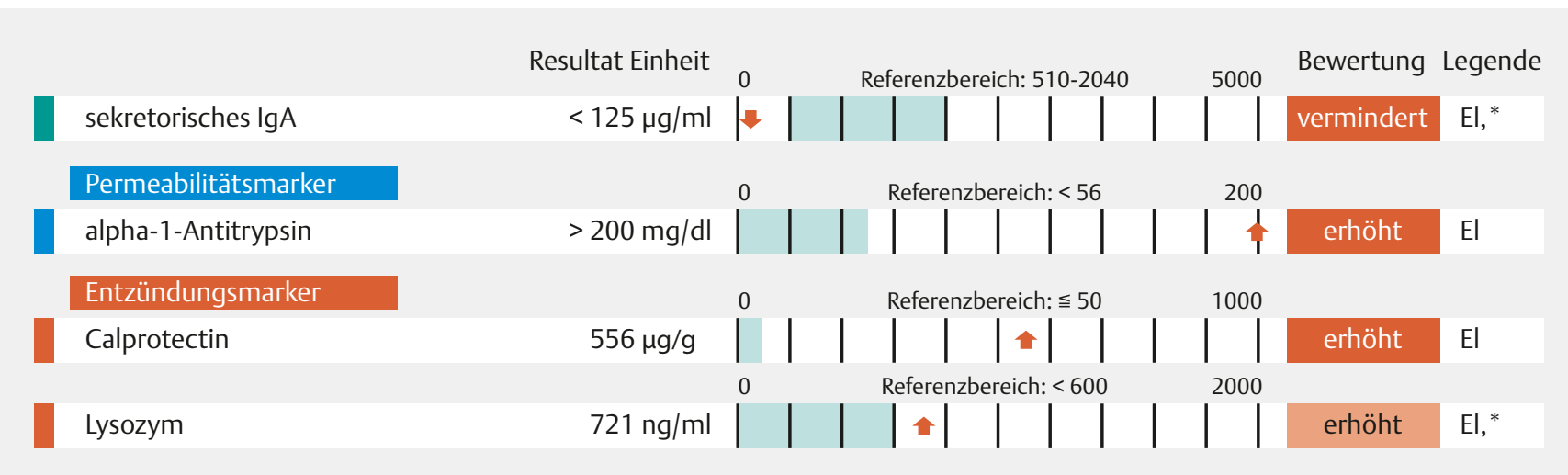

>Abb. 2 Mekoniumanalyse mit deutlichen Anzeichen entzündlicher Schleimhautaktivität.

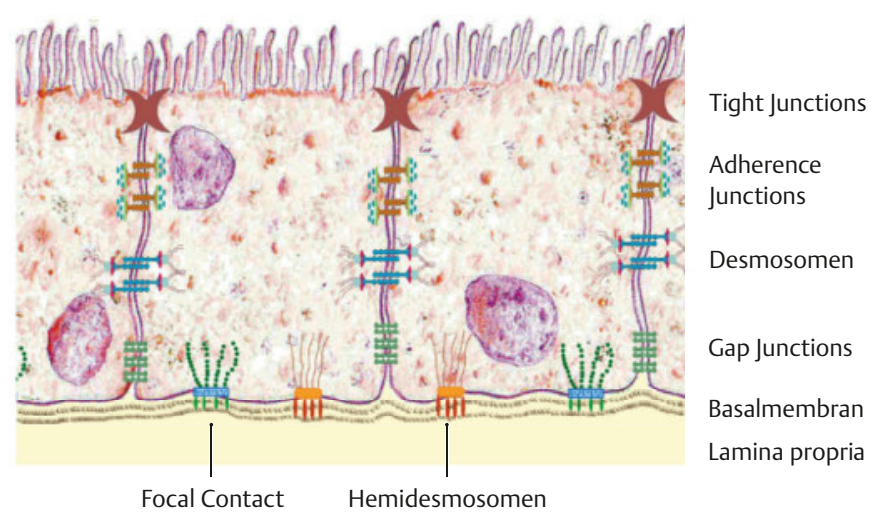

-Abb. 3 Epithelialer Zellverband mit verschiedenen Haftkomplexen. (c) Angèla Ellwanger

hier als erstes an die Einflüsse der Ernährung mit konventioneller Kost gedacht werden: Die Grundnahrungsmittel der „westlichen“ Kost stellen zwei Lebensmittel dar, die für unseren Organismus auf mehreren Ebenen hochproblematisch sind: Kuhmilchprodukte sowie Lebensmittel aus glutenhaltigen Getreidesorten. Bestimmte Inhaltsstoffe wirken unter bestimmten Voraussetzungen proinflammatorisch (Getreidesorten enthalten z. B. verschiedene pflanzeneigene „Fraßgifte“, die aufgrund züchterischer Einflüsse mittlerweile vermehrt im Mehl vorhanden sind [4][5][6][7][9][12]).

\section{Intrauterine mikrobielle Besiedlung}

Die mikrobielle Besiedlung eines Kindes beginnt bereits intrauterin. Über die Schleimhaut gelangen mikrobielle, immunprägende Elemente zum MIS, dann via Plazenta ins Fruchtwasser. Dort werden sie aufgenommen und bereiten das kindliche Immunsystem auf die Umwelt vor. Daneben modulieren direkte immunologische Signale (z. B. regulative T-Zellen, Antikörper, Exosome mit spezifischer MikroRNA) über das Nabelschnurblut die Balance des Immunsystems. So verfügen Neugeborene normalerweise bereits über einen ausreichenden Schleimhautschutz durch eine mikrobiell durchsetzte Mukusschicht (Biofilm) sowie sekretorisches Immunglobulin A (slgA) und B-Defensin 2. Sie können auf bestimmte Reize sowohl pro- als auch antientzündliche Zytokine bilden. Bei einer Ernährung mit Muttermilch wird die mikrobielle Besiedlung durch über 600 verschiedene physiologische Mikroorganismen in der Muttermilch sowie die immunologische Prägung durch mütterliche immunologische Signale (s. o.) fortgesetzt. Durch die Zufuhr von mütterlichem slgA sind gestillte Säuglinge zusätzlich vor Umwelteinflüssen, die das kindliche Immunsystem in dieser Phase noch überfordern würden, geschützt.

Bei einem unkomplizierten Schwangerschafts- und Geburtsverlauf sind Kinder also bestens auf die verschiedensten Umwelteinflüsse vorbereitet. Leidet die Mutter hingegen z. B. an einem chronisch-entzündlichen Krankheitsbild, unter Komplikationen in der Schwangerschaft, besteht eine Dysbiose oder werden in der Schwangerschaft oder Stillzeit chemisch-pharmazeutische Arzneimittel, insbesondere Antibiotika, eingesetzt, hat das einerseits direkt einen erheblichen negativen Einfluss auf die Entwicklung des kindlichen Immunsystems, andererseits aber auch auf die Entwicklung des Milieus und der Grenzfläche sowie der Funktionsfähigkeit und Integrität des Schleimhautorgans mit anhängendem MIS. Stuhlanalysen Neugeborener (Mekoniumanalysen) zeigten in solchen Fällen bereits stark erhöhte schleimhautassoziierte Entzündungsmarker (z. B. Calprotectin, Lysozym, Histamin), die auf vermehrte entzündliche Aktivitäten im kindlichen Schleimhautorgan schließen lassen (• Abb. 2).

Diese Kinder wurden bereits mit einem „brennenden Darm" geboren. Schon physiologischerweise sind die interepithelialen Haftkomplexe (syn. Tight Junctions) beim Neugeborenen noch nicht geschlossen. Besteht zusätzlich bereits bei der Geburt eine entzündliche Aktivität, wird dadurch auch die Entwicklung der mukosalen Barrierefunktion erheblich erschwert und verzögert ( $\triangleright$ Abb. $\mathbf{3})$.

Wird der Säugling zudem dann mit kuhmilchbasierter Säuglingsnahrung gefüttert, kommt es u. a. zu einem un- 
kontrollierten Einstrom von Fremdproteinen in die Lamina propria. Eine immunologische Abwehrreaktion, die primär von den zahlreichen subepithelial liegenden Mastzellen ausgeht, ist die unweigerliche Folge. Es werden vermehrt Histamin, aber auch andere Mediatoren wie Heparin, Zytokine, Tryptasen, Serotonin u. a. freigesetzt, was wiederum entzündlichen Stress an der Schleimhaut bedeutet. Im Rahmen dieser primär dem angeborenen Immunsystem (Th1) zugeschriebenen Abwehrreaktion kommt es im weiteren Verlauf auch zur Bildung von Antikörpern, zunächst der Klasse IgG 1-3. Das kindliche Immunsystem wird damit also bereits am Lebensanfang in eine Abwehrsituation gedrängt, die der Wahrung und Wiederherstellung seiner Integrität dienen soll. Wirft man die genannten weiteren Einflussfaktoren sowie die dadurch zwangsläufig unphysiologische Entwicklung der Säuglingsmikrobiota mit in die Waagschale, wird klar, dass der Organismus unter Umständen diese Situation nicht mehr ausgleichen kann.

\section{Entwicklung einer Allergie}

Mit diesen Überlegungen zum Lebensanfang wird deutlich, wie wichtig eine ungestörte Schwangerschaft, eine natürliche Geburt und eine "artgerechte“ Ernährung sind. Blickt man auf eine sich entwickelnde allergische Reaktion, kann in dieser Zeit völlig unabhängig von genetischen Vorgaben bereits der Grundstein für eine "Allergikerkarriere“ gelegt werden. Die beschriebenen Betrachtungen verdeutlichen, dass die ursprüngliche Bedeutung einer allergischen Reaktion die Initiierung einer Entzündung zum Schutze des Körpers darstellt. Es sind prinzipielle, sinnvolle Formen einer gezielten Abwehr, die vor allem gegen Pathogene, bakterielle Endotoxine oder andere Fremdantigene gerichtet sind. Der Ablauf dieser Entzündungsreaktionen ist normalerweise sehr differenziert reguliert und endet, sobald das auslösende Allergen abgewehrt, unschädlich gemacht und wieder ausgeschieden ist. Eine stetig wachsende Anzahl von Menschen verharrt jedoch in einer solchen Entzündungssituation. Der Grund ist plausibel: Die permanente Abwehrsituation an der Grenzfäche ändert sich nicht. Durch Fehlernährung, Dysbiose und andere Einflüsse (auch Stress, Medikamente) ist bei unserer Lebensweise die Integrität der Grenzfläche permanent in Gefahr. Der normale Verlauf einer entzündlichen Abwehrreaktion scheint somit unterbrochen und kann nicht zu seinem Ende finden. Im Weiteren kommt es schließlich zum bekannten „shift“ der immunologischen Reaktion: Der adaptive Schenkel des Immunsystems, Th2, „übernimmt“ und ab jetzt kommt es zu typischen allergischen Reaktionen vom Typ I, der allergischen Sofortreaktion, deren Klinik von histamininduzierten Symptomen geprägt ist. Die Fähigkeit zur Toleranz gegenüber Umweltantigenen geht immer weiter verloren. In diesem Stadium könnte man von einer „Entzündungsstarre“, aus der der Körper kaum mehr herausfindet, sprechen. Bedenkt man die geschilderten Zusammenhänge und dabei insbesondere die zentra- 
le Bedeutung der Schleimhautintegrität und Mikrobiota, ist bei allergischen Reaktionen die Möglichkeit eines kausalen Therapieansatzes mit den Prinzipien der Mikrobiologischen Therapie einleuchtend und sinnvoll.

\section{Diagnostisches Vorgehen}

In der konventionellen allergologischen Diagnostik stellt die Bestimmung des IgE im Serum das Standardverfahren dar, um eine allergische Reaktion zu objektivieren und einzugrenzen. Es sagt jedoch nichts über die Ursache dieser Entwicklung aus. Die Höhe des IgE-Titers korreliert außerdem oft nicht mit der Schwere der klinischen Symptomatik. Auch persistieren unter erfolgreicher Mikrobiologischer Therapie die hohen IgE-Titer noch eine Zeit lang (ehe sie dann fallen), obwohl der Patient bereits beschwerdefrei ist. Bei allergisch reagierenden Kindern liegt der IgE-Titer in den frühen Krankheitsphasen in den meisten Fällen noch im Normbereich. Erst im weiteren Verlauf wird ein Anstieg beobachtet. Diese Beobachtungen lassen u. a. die Vermutung zu, dass die Hypothese, die Typ-1-allergische Reaktion werde durch die lgE-Antikörper ausgelöst, revidiert werden muss. Möglicherweise kommt dem IgE im Zusammenhang mit der allergischen Reaktion eine andere biologische Bedeutung zu. Der Titeranstieg des IgE ist nach Ansicht der Autoren zunächst nur als Ausdruck einer entgleisten, überschießenden Immunreaktion zu bewerten.

Zur Einschätzung der zugrunde liegenden immunologischen Situation und entsprechenden Planung des therapeutischen Vorgehens ist es demgegenüber vernünftig, sich ein Bild vom Zustand des Schleimhautorgans zu machen: Hier bieten sich validierte Untersuchungsparameter an, die in den Faeces nachweisbar sind. Sie ermöglichen Aussagen zur Funktionalität dieser großen inneren Grenzfläche zur Umwelt. Es lassen sich z. B. Hinweise finden auf:

- Permeabilitätsstörungen (engl. leaky-gut-syndrome): Alpha-1-Antitrypsin, Zonulin,

- verminderte Menge an epithelialen Schutzfaktoren an der Schleimhautoberfläche und damit auch

- Hinweise auf eine Funktionsstörung der Epithelien, v. a. der Enterozyten (z. B. slgA, ß-Defensin 2),

- Aktivitätssteigerung der Mastzellen (Histamin im Stuhl),

- eosinophile und granulozytäre Entzündungsreaktionen (Calprotectin, Lysozym, Laktoferrin, EPX [9][10]).

Ein Nachweis dieser Parameter im Serum gelingt nicht oder korreliert nur bedingt mit der Situation an der Schleimhaut, da die Epithelschicht und das unmittelbar angrenzende subepitheliale Gewebe nicht hämatogen versorgt werden. Die Energieversorgung der Epithelzellen wird durch kurzkettige Fettsäuren aus dem Stoffwechsel bestimmter Mikroorganismen, der sogenannten mukonutritiven Mikrobiota (Faecalibacterium prausnitzii, Akkermansia muciniphila) bereitgestellt. Durch Bestimmung der Buttersäure im Stuhl, der kurzkettigen Fettsäure, die eine
Hauptenergiequelle der Enterozyten darstellt, kann auch der „Ernährungszustand“ der Enterozyten abgeschätzt werden. Zeitgleich können aus ein- und derselben Stuhlprobe auch die Zusammensetzung der Mikrobiota (früher Darmflora) bestimmt sowie Hinweise auf das intestinale Milieu und die Verdauungsleistung des Organismus gewonnen werden.

Mit Blick auf den Zustand des Schleimhautorgans und möglicher Belastungen stellt neben den oben genannten Parametern die serologische Untersuchung von IgG13-Antikörpern gegenüber Lebensmitteln eine sinnvolle Maßnahme dar. Bei einer Integritätsstörung des Schleimhautorgans, formal leaky-gut-syndrome, gelangt Darminhalt unkontrolliert in die Submukosa und löst somit eine Entzündungsreaktion aus. Diese richtet sich in erster Linie gegen die eingedrungenen Mikroorganismen, da von ihnen die größte Gefahr für den Organismus ausgeht (Infektion). Die "begleitende“ Bildung von Antikörpern auch gegenüber Lebensmitteln muss therapeutisch unbedingt beachtet werden: Bei jedem Verzehr der betroffenen Lebensmittel kommt es zu einer entsprechenden Immunreaktion mit Anfall von Immunkomplexen an der Schleimhaut, die phagozytiert werden müssen. Diese können, insbesondere bei eingeschränkter mukosaler Barrierefunktion, auch in den submukosalen Raum und via MIS ins Körperinnere gelangen. Um diese Entzündungsreaktion zu vermeiden, müssen positiv getestete Lebensmittel für einige Zeit gemieden werden.

Je mehr potentiell schleimhautschädigende oder milieubelastende Einflüsse herausgefunden werden, desto effektiver kann durch deren Reduktion oder gezielte Behandlung im Rahmen des therapeutischen Konzeptes zur Entlastung der Schleimhaut und Wiederherstellung der Schleimhautintegrität beigetragen werden. Dazu zählt im Einzelfall auch die genaue anamnestische Erfragung der Ernährungsgewohnheiten und insbesondere „begleitender" abdomineller Probleme zur Erfassung von Intoleranzen oder pseudoallergischen Reaktionen.

\section{Therapie der allergischen Reaktion}

Im Mittelpunkt einer kausalen Behandlung der allergischen Reaktion steht die Mikrobiologische Therapie.

Bisher sind folgende Wirkungen der Therapie mit Probiotika bekannt:

- Verbesserung eines gestörten Milieus an der Schleimhaut (z. B. infolge einer Fehlernährung, Maldigestion bei einer Unterversorgung an Verdauungsfermenten, mikrobielle Mangel- oder Fehlbesiedlung infolge wiederholter antibiotischer oder anderer chemisch-pharmazeutischer Therapien, Freisetzung von Endotoxinen mikrobiellen Ursprungs etc.)

- Optimierung der Nährstoffversorgung von Enterozyten 
- Verbesserung von Kolonisationsresistenz und Milieustabilität durch

- Adhäsionskonkurrenz zu Pathogenen am Schleimhautepithel

- Synthese antimikrobieller Substanzen (z. B. Defensine, Bakteriozine)

- Inaktivierung von Toxinen

- Beeinflussung der mikrobiellen Balance bei einer Vielzahl von immunmodulatorischen und stoffwechselbezogenen Einflüssen (Informationshomöostase)

- Beeinflussung von enterozytären und neuronalen Signalen des ENS (z. B. Transmitterausschüttung und Reagibilitätsverhalten, Darmmotilität, vagale Signalweiterleitung, Darm-Hirn-Achse u.v.m)

- Milieumodulation und -stabilisierung durch

- Beeinflussung des „quorum sensing“ und „crosstalk“

- Verbesserung der Biofilmeigenschaften (mukonutritive Mikrobiota)

- antiinflammatorische Signale: Herunterregulation proinflammatorischer Signalmoleküle, Modifikation der TLR-Regulation

- Stabilisierung der enterozytären Barrierefunktion

- Verbesserung der Immunfunktionen (z. B. Steigerung der slgA- und ß-Defensinproduktion u.v.m)

Der Aufbau des Therapiekonzeptes richtet sich nach der Klinik, Art und Ausprägung einer bestehenden Dysbiose, entzündlichen Schleimhautreaktion oder Permeabilitätssteigerung. Zunächst sollte eine Milieuentlastung und Beruhigung entzündlicher Schleimhautvorgänge angestrebt werden. Dabei kommen Bakterienpräparate der Protektivmikrobiota (Laktobazillen, Bifidobakterien) sowie Adsorber (Kohle, Zeolithe, Löß), Phytotherapeutika (Myrrhe, Kamille, Weihrauch) und Homöopathika (Lymphomyosot ${ }^{\circledR}$, Mucosa comp ${ }^{\circledR}$ ) zum Einsatz. Oft kann man auch bereits Zellwandbestandteile und bakterielle Stoffwechselprodukte der Immunmikrobiota mit einsetzen. Daneben ist das Einhalten einer Karenzdiät positiv getesteter Lebensmit-

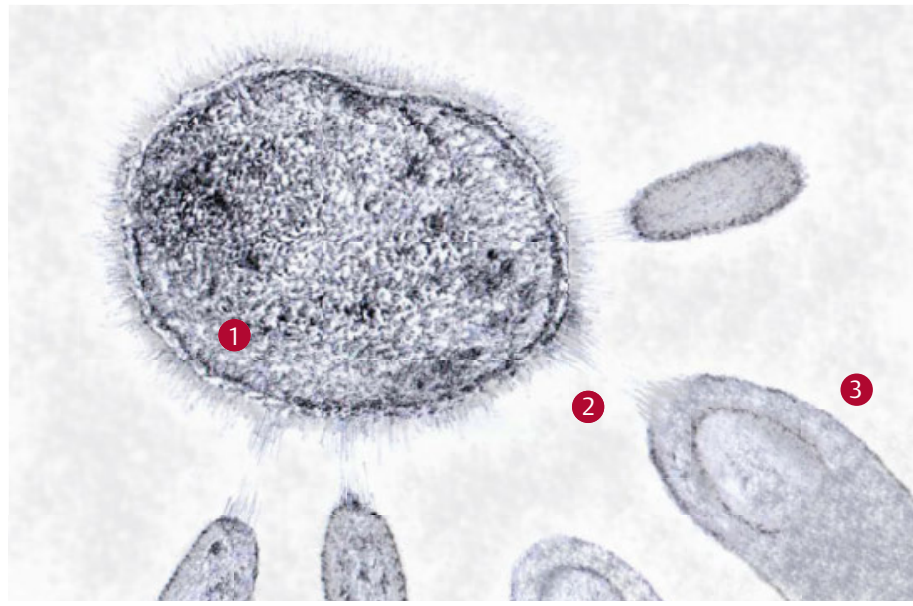

-Abb. 4 Bakterium und Schleimhautassoziation. 1 Bakterium, 2 Crosstalk, 3 Mikrovilli (Enterozyt). @ Angèla Ellwanger

tel (IgG 1-3) unumgänglich. Bei allergisch reagierenden Patienten ist es aus stoffwechselphysiologischen sowie immunologischen Gründen von großer Bedeutung, Kuhmilch- und Getreideprodukte in der Ernährung drastisch zu reduzieren.

Im Weiteren wird die Mikrobiologische Therapie je nach Entwicklung des klinischen Bildes modifiziert und intensiviert. Bereits mit den bisher eingesetzten Probiotika kommt es neben dem verbesserten Schleimhautschutz zu einer Vielzahl immunologischer Einflüsse im Milieu und Immunsystem. Die oben angegebenen Effekte der Mikrobiologischen Therapie sind natürlich nicht einzeln anzugehen - die Reaktionen und Regulationen greifen ineinander und geschehen parallel. Generell setzen alle therapeutisch verabreichten Mikroorganismen und deren Bestandteile immunologische Signale ( $\triangleright$ Abb. 4).

Nach Wiederherstellung der Schleimhautintegrität und Stabilisierung der Verhältnisse im Grenzraum können durch den bewussten Einsatz der sogenannten Immun- 
bakterien (apathogene Enterococcus faecalis und E.-coliSpezies) sehr gut gezielte immunmodulatorische Effekte angestoßen werden. Mit Auto-Coli-Vaccinen aus den eigenen E.-Coli-Bakterien der Patienten werden spezifisch individuelle immunregulierende Wirkungen erzielt. Der Einsatz der Autovaccinetherapie kann nach erfolgreicher Stabilisierung der Schleimhaut und des Milieus beginnen, wenn sich das MIS bereits "beruhigt" hat.

Die Behandlungsdauer mit Mikrobiologischer Therapie richtet sich nach dem Beschwerdebild und den initialen Schleimhautdysfunktionen. Es sollte mit einer Behandlungsdauer von 6-12 Monaten gerechnet werden, manchmal dauert die Behandlung auch länger.

\section{Prävention}

Es ist naheliegend, diese Therapieansätze auch präventiv zu nutzen, denn es geht nicht nur um allergische Reaktionen. Inzwischen wissen wir, dass die Verhältnisse im Grenzraum und im mikrobiellen Milieu, „chronic silent inflammation “, sowie bakterielle Stoffwechselprodukte für eine Vielzahl von Krankheitsbildern mit verantwortlich sind. Eine Mikrobiologische Therapie der Mutter während der Schwangerschaft und Stillzeit oder die Versorgung von Neugeborenen (gerade von Kindern, die per Kaiserschnitt entbunden wurden) mit Bakterien der Protektivmikrobiota ist eine vielversprechende Möglichkeit, eine normale Immunogenese zu ermöglichen [2][3][8]. Eine begleitende Mikrobiologische Therapie z. B. bei notwendigen antibiotischen Behandlungen wird inzwischen bereits häufiger beachtet und kann der Entwicklung einer Dysbiose sowie dem entsprechenden Fehlen wichtiger mikrobieller Signale wirkungsvoll entgegenwirken. Sinnvoll ist es, bereits bei ersten Anzeichen von Milieustörungen an eine Mikrobiologische Therapie zu denken und damit einer möglichen Entwicklung allergischer oder anderer chronischentzündlicher Krankheitsbilder frühzeitig therapeutisch zu begegnen [9][11].

\section{Interessenkonflikt}

Die Autoren sind als Referenten für das Institut für Mikroökologie tätig.

\section{Autoren}

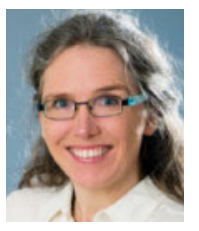

\section{Dr. med. Susanne Schnitzer}

Susanne Schnitzer ist Ärztin für Innere Medizin mit den Schwerpunkten „Biologische Medizin“ und „Scenartherapie“, seit 2013 Niederlassung in eigener Praxis. Sie befasst sich intensiv mit der menschlichen Mikroökologie und vertritt ein systemisches Krankheitsverständnis. Sie ist als Referentin für den Arbeitskreis für Mikrobiologische Therapie e.V. tätig.

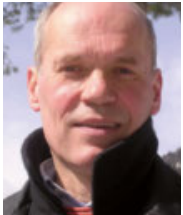

\section{Dr. med. Rainer Schmidt}

Rainer Schmidt ist Arzt für Kinder- und Jugendmedizin mit den Zusatzbezeichnungen Allergologie und Naturheilverfahren. $\mathrm{Er}$ arbeitet schwerpunktmäßig mit der Mikrobiologischen Therapie und anderen Naturheilverfahren und ist zudem als Referent des Arbeitskreises für Mikrobiologische Therapie e. V. tätig.

\section{Korrespondenzadresse}

\author{
Dr. med. Susanne Schnitzer \\ Höhenweg 4 \\ 91094 Bräuningshof \\ Tel. 091337679670 \\ E-Mail: allergie_mikrobiota@schnitzer.emai
}

\section{Literatur}

[1] Arrieta MC et al. Early infancy microbial and metabolic alterations affect risk of childhood asthma. Sci Transl Med 2015; 7 (307): 307ra152

[2] Cuello-Garcia CA. Probiotics for the prevention of allergy: A systematic review and meta-analysis of randomized controlled trials. J Allergy Clin Immunol 2015; 136 (4): 952-961

[3] Lau $S$ et al. Oral application of bacterial lysate in infancy decreases the risk of atopic dermatitis in children with 1 atopic parent in a randomized, placebo-controlled trial. J Allergy Clin Immunol 2012; 129: 1040-1047

[4] Melnik BC. Milk - A nutrient system of mammalian evolution promoting mTORC1-dependent translation. Int J Mol Sci 2015; 16: 17048-17087

[5] Melnik BC. The potential mechanistic link between allergy and obesity development and infant formula feeding. Allergy Asthma Clin Immunol 2014; 10: 37

[6] Melnik BC et al. Milk is not just food but most likely a genetic transfection system activating mTORC1 signaling for postnatal growth. Nutr J 2013; 12: 103

[7] Melnik BC et al. Milk: the promotor of chronic modern Western diseases. Med Hypotheses 2009; 72: 631-639

[8] Rautava et al. Maternal probiotic supplementation during pregnancy and breast-feeding reduces the risk of eczema in the infant. Allergy Clin Immunol 2012; 130 (6): 1355-1360

[9] Schmidt R, Schnitzer S. Allergie und Mikrobiota. Stuttgart: Haug; 2017

[10] Schmidt R. Wie die Schleimhaut „spricht“ - Stuhlanalytik bei schleimhautassoziierten Krankheitsbildern. OM \& Ernährung 2012; 140: 2-10

[11] Schmidt R. Das allergiekranke Kind - Mikroorganismen in der Diskussion. Erfahrungsheilkunde 2008, 57: 104-108

[12] Schnitzer S. Bestandteile glutenhaltiger Getreidesorten und ihr Einfluss auf Stoffwechsel und Immunsystem, Grenzfläche und Milieu. OM \& Ernährung 2016; 157: 30-36

\section{Bibliografie}

DOI https://doi.org/10.1055/s-0043-124285

EHK 2018; 67: 6-12

(c) MVS Medizinverlage Stuttgart GmbH \& Co. KG ISSN 0014-0082 